\title{
A decommissioned LHC model magnet as an axion telescope
}

K. Zioutas ${ }^{1}$, C.E. Aalseth ${ }^{2}$, D. Abriola ${ }^{3}$, F.T. Avignone III ${ }^{2}$, R.L. Brodzinski ${ }^{4}$, J.I. Collar ${ }^{*}$, R. Creswick ${ }^{2}$, D.E. Di Gregorio ${ }^{3}$, H. Farach ${ }^{2}$, A.O. Gattone ${ }^{3}$, C.K. Guérard ${ }^{3}$, F. Hasenbalg ${ }^{3}$, M. Hasinoff ${ }^{6}$, H. Huck ${ }^{3}$, A. Liolios ${ }^{1}$, H.S. Miley ${ }^{4}$, A. Morales ${ }^{7}$, J. Morales ${ }^{7}$, D. Nikas ${ }^{1}$, S. Nussinov ${ }^{8}$, A. Ortiz ${ }^{7}$, E. Savvidis ${ }^{1}$, S. Scopel ${ }^{7}$, P. Sievers ${ }^{5}$, J.A. Villar ${ }^{7}$, L. Walckiers ${ }^{5}$.

1) Physics Department, University of Thessaloniki, GR-54006 Thessaloniki, Greece.

2) Department of Physics and Astronomy, University of South Carolina, Columbia, SC 29208, USA.

3) Department of Physics, TANDAR Laboratory, C.N.E.A., Buenos Aires, Argentina.

4) Pacific Northwest National Laboratory, Richland, WA 99352, USA.

5) $C E R N, C H-1221$ Geneva 23, Switzerland.

6) Department of Physics and Astronomy, University of British Columbia, Vancouver BC V6T IZI, Canada.

7) Laboratorio de Fisica Nuclear y Atlas Energias, Faculdad de Ciencias, Universidad de Zaragoza, E50009 Zaragoza, Spain.

8) Department of Physics, Tel Aviv University, Tel Aviv, Israel.

\begin{abstract}
The 8.4 Tesla, $10 \mathrm{~m}$ long transverse magnetic field of a twin aperture LHC bending magnet can be utilized as a macroscopic coherent solar axion-to-photon converter. Numerical calculations show that the integrated time of alignment with the Sun would be 33 days per year with the magnet on a tracking table capable of $\pm 5^{\circ}$ in the vertical direction and $\pm 40^{\circ}$ in the horizontal direction. The existing lower bound on the axion-to-photon coupling constant can be improved by a factor between 30 and 100 in 3 years, i.e., $g_{a \gamma \gamma} \lesssim 9 \cdot 10^{-11} \mathrm{GeV}^{-1}$ for axion masses $\lesssim 1 \mathrm{eV}$. This value falls within the existing open axion mass window. The same set-up can simultaneously search for lowand high-energy celestial axions, or axion-like particles, scanning the sky as the Earth rotates and orbits the Sun.
\end{abstract}

PASC numbers: 41.85.Lc, 85.25.Ly, 14.80.-j, 14.80.Mz, 92.60.Vb, 95.

Keywords: (Solar) Axions, LHC, (Superconducting) Magnets, Dark Matter.

*) E-mail : JUAN.COLLAR@CERN.CH

Proofs should be sent to: J.I. Collar (address above), phone: +331 44278237, fax: +331 43542878, collar@mail.cern.ch 


\section{INTRODUCTION}

QCD, the universally accepted theory of strong interactions has one serious blemish, the $\mathrm{U}(1)$ problem (why the $\eta^{\prime} / \pi$ mass ratio is not closer to unity [1]). Its solution produces in turn the strong CP problem (why the neutron's electric dipole moment is at least a factor $10^{-9}$ smaller than expected). An attractive solution to this invokes a new $\mathrm{U}_{P Q}(1)$ symmetry, the Peccei-Quinn symmetry. The spontaneous breaking of this new symmetry predicts the existence of a light neutral pseudoscalar particle, the axion, which is closely related to the neutral pion [2,3]. The axion also arises in supersymmetry and superstring theories. In addition, axions are one of the most interesting non-baryonic candidates for the ubiquitous dark matter (DM) [4 [7]. Axions may also exist as primordial cosmic relics copiously produced in the early Universe. For these reasons, axions have received much attention. More details about axions and their role in cosmology and astrophysics can be found in [8,9]. A remarkably pedagogical introduction to them is given by Sikivie in [10].

The search for relic axions is based mainly on the Primakoff effect and is performed with superconducting resonant cavities 111] or, also recently, with CERN's SMC polarized target [14]. Fig. 1, taken from ref. [14], shows the present status for axion searches. The main limitation of the sensitive experiments described in [11 [13] is the possibility that the axion rest mass lies outside the resonance region of the micro-wave cavities utilized. However, energetic axions might also be created continuously in reactions taking place in red giants, supernovae, and in particular inside our Sun, this being the nearest and brightest potential axion source in the $1-15 \mathrm{keV}$ total energy range. Experiments searching for solar axions have the main advantage of being sensitive in principle to a very broad axion rest mass range $\left(m_{a} c^{2} \lesssim 10 \mathrm{keV}\right)$, although coherence-loss constraints decrease the sensitivity for axion masses above $\sim 1 \mathrm{eV}[6]$.

Astrophysical considerations (cooling rates of stars), cosmological arguments (overclosure of the Universe), and laboratory experiments require an axion mass in the range [5, 15,18

$$
10^{-5} e V<m_{a} c^{2}<10^{-3} e V
$$

while the mass range

$$
2 \mathrm{eV}<m_{a} c^{2}<10 \mathrm{eV}
$$

is still possible for hadronic axions with couplings to leptons equal to zero [6, 19].

The axion decay into two photons $(a \rightarrow \gamma \gamma)$ is the reaction mainly used to search for axions. Inside a magnetic field, the axion couples to a virtual photon $\left(\gamma_{\text {virtual }}\right)$, producing a real photon $(\gamma)$ via the Primakoff effect. This real photon can then be detected :

$$
a+\gamma_{\text {virtual }} \rightleftharpoons \gamma
$$

The energy of this photon $(\gamma)$ is then equal to the axion's total energy. The average energy of the emitted solar axions, and therefore of the converted photons is $\sim 4.2 \mathrm{keV}$ 
[20]. In principle, reaction (3) allows for (coherent) photon mixing with spin 0 , 1 , or 2 particles (e.g. spin-0 for axion and spin-2 for the graviton [21]). An improvement in the axion-to-photon coupling limit implies an equivalent improvement in the axion mass limit; these quantities are related by 22]

$$
g_{a \gamma \gamma} \equiv M^{-1} \approx 10^{-10}\left[\frac{m_{a}}{1 e V}\right] G e V^{-1},
$$

where $M$ is the corresponding mass scale of the spontaneously broken new symmetry $\left(\mathrm{U}_{P Q}(1)\right)$. Models of hadronic axions with enhanced or supressed photon couplings are easily constructed [23]; here the factor in front of $m_{a}$ in Eq. (4) is chosen to vary within an indicative range $10^{-9}-10^{-11}$, to form the broad band labeled "axion models" in Figs. 1,3 .

In this paper it is shown that the first twin-aperture superconducting LHC prototype magnets at CERN have an interesting application as a solar axion detector, without interfering with the LHC construction (these are test magnets and will become available for this independent experiment at the end of 1998. A suitable experimental hall to house the set-up will be required). The general experimental concept is to have the magnet aligned with the Sun for a period as long as possible, using an active tracking system; the Primakoff converted photons, emitted in the direction of the incoming axion and carrying its original energy and momentum, are then detected by ultralow-background detectors at the end of the magnetic pipe lines. A hypothetical axion signal is then expected only during the times of solar alignment with the magnet axis, providing a unique signature. The suggested axion telescope can also discover or exclude other hypothetical (axion-like) celestial particles with similar couplings, with energies above $\sim$ few $\mathrm{keV}$.

\section{AXION-TO-PHOTON CONVERSION}

An experimental limit for the axion-to-photon coupling inside a magnetic field was obtained in 1992 by the pioneering axion experiment of the Rochester-BNL-FNAL collaboration [24] :

$g_{a \gamma \gamma}<3.6 \cdot 10^{-9} \mathrm{GeV}^{-1}$ for $m_{a}<0.03 \mathrm{eV}$ and $g_{a \gamma \gamma} \lesssim 7.7 \cdot 10^{-9} \mathrm{GeV}^{-1}$ for $0.03<m_{a} \lesssim 0.1 \mathrm{eV}$

Most of the theoretical relations and the expected experimental parameters of relevance are taken from refs. [20,21,24,25]. The magnetic field strength (B) and its length (L) are the fundamental parameters in the calculation of the performance of a detector to be used as a coherent axion-to-photon converter. The probability of detecting a photon in the $\sim 1-15 \mathrm{keV}$ region, per solar axion entering one of the magnetic field pipes, is

$$
P_{a \rightarrow \gamma}=\left[\frac{\mathrm{B} \cdot \mathrm{L}}{2 M}\right]^{2},
$$


assuming $100 \%$ detection efficiency for the conversion x-rays.

The conversion probability is :

$$
P_{a \rightarrow \gamma} \approx 1.8 \times 10^{-17} \times\left(\left[\frac{\mathrm{B}}{8.4 T}\right]^{2} \cdot\left[\frac{\mathrm{L}}{10 m}\right]^{2} \cdot\left[\frac{10^{10} \mathrm{GeV}}{M}\right]^{2}\right)
$$

(with $\left.g_{a \gamma \gamma} \equiv 1 / M\right)$.

On Earth, the total solar axion flux $\Phi_{a}$ is [20] :

$$
\Phi_{a} \approx 3.5 \cdot 10^{11}\left[\frac{10^{10} \mathrm{GeV}}{M}\right]^{2} / \mathrm{cm}^{2} \cdot \mathrm{s}
$$

It follows that the integrated axion flux $\Phi_{a}^{L H C}$ crossing an LHC bending magnet (with a cross-section area $F \approx 2 \times 14 \mathrm{~cm}^{2}$ ), while being aligned with the Sun's core, is :

$$
\Phi_{a}^{L H C} \approx 8.6 \times 10^{17}\left[\frac{10^{10} \mathrm{GeV}}{M}\right]^{2} \text { axions } / d .
$$

Combining the axion conversion efficiency (Eq. (7), evaluated at the indicated fiducial values of $B, L$ ) with the axion fluence (Eq. (9)), the estimated number $(\mathrm{R})$ of $\mathrm{x}$-rays due to the converted axions for a solar alignment time of $24 h$ within 1 year, attainable with just $\mathrm{a} \pm 1.5^{\circ}$ tracking along the horizontal and vertical axis (Fig. 2), is

$$
R \approx 1.8 \cdot 10^{-17} \times 8.6 \cdot 10^{17} \cdot\left[\frac{10^{10} \mathrm{GeV}}{M}\right]^{4}=15 \text { events } /(24 h \text { per year }),
$$

given $M=10^{10} \mathrm{GeV}$. Taking into account the estimated background in the x-ray detectors (Eq. (14)-(15)), discussed below, a value for the coupling constant $g_{a \gamma \gamma}>$ $10^{-10} \mathrm{GeV}^{-1}$ is then expected to be rejected at the $3 \sigma$ level within the first 2 years, even with this modest $\pm 1.5^{\circ}$ tracking. This limit would be better by a factor $\sim 30$ than that obtained in ref. 24]. In order to show the maximum improvement reachable with only one LHC model bending magnet, these figures can be scaled up. Let us take 2 years of measuring time, $\pm 40^{\circ}$ horizontal and $\pm 5^{\circ}$ vertical tracking ( $=33$ days of integrated solar-alignment time per year, Fig. 2). Relation (10) then yields

$$
R \approx 195 \text { events/(66 days in } 2 \text { years }),
$$

when $M=1.51 \cdot 10^{10} \mathrm{GeV}\left(g_{a \gamma \gamma}=6.6 \cdot 10^{-11} \mathrm{GeV}^{-1}\right)$ (Fig. 3). This rate is roughly three times the statistical fluctuation in the background estimated in Eq. (15). Therefore, the new limit on the axion-to-photon coupling constant, at the $\sim 3 \sigma$ level, and for comparison with ref. [24], would be

$$
g_{a \gamma \gamma}<6.6 \cdot 10^{-11} \mathrm{GeV}^{-1} .
$$

This represents an improvement by a factor $\sim 55$. Similarly, considering one single 14 $\mathrm{m}$ bending magnet or two $10 \mathrm{~m}$ magnets in series, the expected limits for the coupling constant $g_{a \gamma \gamma}$ are $5.2 \cdot 10^{-11} \mathrm{GeV}^{-1}$ and $4.6 \cdot 10^{-11} \mathrm{GeV}^{-1}$, respectively. The possible 
improvement is then a factor of 70-80. The $M^{-4}$ dependence of the (solar) axion-tophoton conversion rate (Equation (10)) makes an experiment with further improvement rather difficult.

Coherence : For massive axions, in order to fulfill the coherence relation (6), i.e. to avoid deconstructive axion-photon interference, the magnetic field length $(L)$ must be 24] :

$$
L<\frac{(2 \pi \hbar c) \cdot(\hbar \omega)}{m_{a}^{2} c^{4}}
$$

For example, a coherence length of $L=10-20 \mathrm{~m}$ in vacuum requires $m_{a} \leq 0.01 \mathrm{eV}$ for a photon energy $\hbar \omega \approx 5 \mathrm{keV}$; this condition is fulfilled when the x-ray detector is placed outside the magnet (see ref. [24]). Note that according to relation (13), axions of higher energy have an accordingly longer coherent conversion pathlength. Thus, 511 $\mathrm{keV}$ and $\sim 60 \mathrm{MeV}$ axions, which could be produced during positron annihilation and supernovae explosions [22, can be similarly detected with $L \sim 10 \mathrm{~m}$ magnets even if they have a rest mass of up to 0.1 or $1 \mathrm{eV}$, respectively. In other words, such a powerful axion-to-photon converter (combined with a high-efficiency, low-background detector), can be used to simultaneously search for low- as well as high-energy celestial axions; the active anti-Compton shielding (see below) can be used for this purpose too. Due to the Earth's motion almost the whole sky can be scanned progressively (Fig. 4).

Coherence can be restored for a solar axion rest mass of up to $\sim 1 \mathrm{eV}$ by filling the magnetic conversion region with buffer gas [20], e.g. He gas at a pressure up to 10 atm, this being the limiting value since the absorption length for $\sim 4 \mathrm{keV}$ photons then approaches $10 \mathrm{~m}$, the magnet length. For an appropriate gas pressure, the 'dressed' photons inside the buffer gas, coming from the converted axions, acquire an effective mass $\left(m_{\gamma}\right)$ whose wavelength can match the axion's wavelength, thus preventing deconstructive interference, i.e., coherence is preserved for a narrow mass window. By limiting ourselves to $<10 \mathrm{~atm}$, no complication is expected due to photon interaction with the buffer gas, whose pressure should be varied in appropriate steps (after the first $\sim 2$ years of measurement in vacuum), in order to cover axion masses above $\sim 0.01 \mathrm{eV}$. In this manner the expected sensitivity on the axion-to-photon coupling constant can reach the theoretically favoured regions above $\sim 0.01 \mathrm{eV}$ (Fig. 1,3). The operating pressure (at $\left.300^{\circ} \mathrm{K}\right)$ is $P[\mathrm{~atm}] \approx 15 \cdot m_{\gamma}[\mathrm{eV}]$ for a chosen 'photon mass', $m_{\gamma}$ 20]. This type of measurements will require an additional 1-2 years to be completed, in order to measure several points in the range $0.02 \leq m_{a} \leq 1 \mathrm{eV}$ and $g_{a \gamma \gamma}<10^{-10} \mathrm{GeV}^{-1}$ (the Red Giant limit). However, even with vacuum in the magnetic field pipes, the axion rest mass range that can be investigated still fully covers the allowed region given in (1).

\section{THE PHOTON DETECTOR}

In order to minimize Compton scattering from higher energy background photons, a commercial detector consisting of a thin Ge crystal with an integral Compton suppression 
crystal should be used. A low-background electro-formed $\mathrm{Cu}$ cryostat and $\sim 20 \mathrm{~cm} \mathrm{~Pb}$ shielding surrounded with a $10 \mathrm{~cm}$ plastic scintillator, to veto cosmic-rays, will further suppress the background. Additional moderator outside the plastic would be desirable as well. Special precautions against low-energy microphonics must be taken using wellknown discrimination techniques based on pulse-shape analysis [29]. Thin n-type Ge detectors with a Be window (53 $\mathrm{mm}$ in diameter, $50 \mathrm{~g}$ in weight) manufactured to our specifications are being considered. The expected energy threshold is $\sim 600 \mathrm{eV}$ and the low-energy resolution is FWHM $\approx 400 \mathrm{eV}$. Similar low-background experiments have been performed at ground level by members of this collaboration [30.

Background estimation. The achievable background $(N)$ at sea level for the two Ge x-ray detectors (50 grams each), one at each end of the magnet, is [30,31] :

$$
N \approx 50-80 \text { counts } / \mathrm{d} \cdot \mathrm{kg} \cdot \mathrm{keV} \approx 65 \mathrm{counts} / \mathrm{d} \cdot 2 \times 50 \mathrm{~g} \cdot 10 \mathrm{keV}
$$

(10 keV is the width of the solar axion spectrum).

The experimentally determined background level, for the $\sim 66$ days of integrated exposure to the Sun during the first 2 years, will be

$$
N \approx(4290 \pm 65) \text { counts } / 66 \mathrm{~d} \cdot 2 \times 50 \mathrm{~g} \cdot 10 \mathrm{keV}
$$

However, it is the background fluctuation $( \pm 65)$ that imposes a limit on the maximum allowable number of axion events (Equations (10) and (11)), and not the background level itself. The uncertainty in the average background can be made negligible by running the detectors (with the magnet switched-off) during the times of solar misalignment. There is no immediate advantage in bringing the set-up to an underground location, since the further reduction in background (a factor of $\sim 20$ [32]) translates into an increase in $g_{a \gamma \gamma}$

sensitivity of only $\sim 1.5$ (Eq. 10, 11). This relatively weak dependence $\left(\sim N^{1 / 8}\right)$ of the achievable limits on the detector background may make of PIN diodes an inexpensive alternative to be considered [33, 34].

\section{THE MAGNET}

\subsection{The existing set-up}

First generation twin aperture superconducting LHC magnets have been built and tested [35. Their nominal field is $8.4 \mathrm{~T}$, but they routinely reach fields between 9 and $9.5 \mathrm{~T}$. Their effective magnetic length is $9.25 \mathrm{~m}$. These prototype magnets are not bent to cope with the LHC radius of curvature. They have straight cold bores of $42.5 \mathrm{~mm}$ aperture. All these magnets have been tested and measured magnetically on a bench in the SM18 facility at CERN [36]. Three of the seven magnets tested underwent a thorough life test. A $30 \mathrm{~m}$ long girder is used to support the magnet under test together with the MFB (Magnet Feed Box) housing all the cryogenic and electrical connections needed for the tests in superfluid helium. The girder can be presently tilted about two rotation axes to simulate the possible inclinations of the magnets in the LEP/LHC tunnel $( \pm 1.5 \%$ of slope). The magnetic measurements were performed with rotating coils at room 
temperature by sliding warm fingers inside. These anticryostats have a $35 \mathrm{~mm}$ aperture and a length of $13.8 \mathrm{~m}$ to go through the MFB and have been used opened at both ends or closed and pumped down.

\subsection{Necessary improvements}

The stroke of the jack can be increased, in order to provide an inclination of the girder to $\pm 10^{\circ}$. The shear stress so produced by the magnet's weight in the cryostat's feet is well within acceptable limits. The cross section and robustness of these feet are minimized to reduce thermal losses: a more detailed analysis of the structure, leading to possible reinforcement, must be performed to allow for an even higher inclination range. The operation of the cryostat with superfluid helium is advantageous: the magnet's end opposite to the MFB is still immersed in liquid He when in its highest position.

The cryogenic piping and electrical connections to feed the $15 \mathrm{kA}$ needed can easily be made flexible enough to allow a rotation of the girder about a vertical axis, preferably centered on the MFB, to a range of $\sim \pm 40^{\circ}$.

\section{DISCUSSION}

The axion-to-photon conversion via the Primakoff effect inside the LHC model magnets is the established working principle [6,24 26] of the proposed axion helioscope. The same effect allows for axion production inside celestial objects like our Sun, which translates into exotic energy-loss mechanisms that may determine their evolution. Such an experiment will also be sensitive to the suggested monochromatic $14.4 \mathrm{keV}$ nuclear axion emission from the Sun's core [27]. Besides the speculated role of relic axions as dark matter constituents and their potential ability to solve the strong CP problem, the question of their existence or non-existence becomes of paramount importance with far-reaching consequences for particle (astro)physics and cosmology.

In conclusion, compared with the previously achieved experimental results, the proposed CERN experiment has the following advantages : 1) the active tracking of the Sun is very important because it increases the alignment period by a factor $\approx 35$ (for $\pm 1.5^{\circ}$ tracking) compared with a fixed magnet geometry (see Fig. 2a,b). This period scales with the maximum tracking angle along the horizontal and vertical axis. For comparison, in the suggestive work of ref. [25] the alignment period is only $\approx 30$ sec per year. 2) Solar axion signals are expected to show up at precisely predicted time-windows, displaying a semi-diurnal periodicity, allowing us to exclude systematical uncertainties and providing a unique signature. The expected improvement in the experimental coupling constant $g_{a \gamma \gamma}$ can be as much as a factor 30 to $\sim 50$ within 1 to 3 years for $3 \times 2$ months of exposure, depending on the attainable tracking range. 3) Two twin aperture model magnets in series with $\pm 10^{\circ}$ vertical tracking can provide an improvement by a factor as large as $\sim 100$. 4) With the same detection system, low- as well as high-energy celestial axions, whatever their origin, can be detected simultaneously due to the implicit directionality (coming from the Earth's motion), entering a serendipitous experimental 
realm, that of axion astronomy. For this kind of search, no active tracking is necessary, but is desirable (see Fig. 4).

Because of the associated increase in the mass scale up to $M \simeq 2.5 \cdot 10^{10} \mathrm{GeV}$, this search is sensitive to potentially new physics beyond the Standard Model, taking advantage of the LHC-magnet testing program and facilities.

\section{Acknowledgements}

We would like to thank Y. Semertzidis (BNL) for many informative discussions. 


\section{Figure captions}

\section{Figure 1.}

Limits on the axion coupling constant to two photons $\left(g_{a \gamma \gamma}\right)$ versus its rest mass from laboratory and astrophysical considerations [14]. The theoretically-favoured relation between the axion-to-photon coupling strength and the axion mass is also shown. The recent limit from SN1987A is taken from ref. [28]. The expected limit to be achieved with this proposal $\left(\mathrm{L}=10 \mathrm{~m}, \pm 5^{\circ}\right.$ vertical tracking, $\pm 40^{\circ}$ horizontal) is also shown; the attached hatched region refers to measurements with He gas in the magnetic field pipes. The astrophysical limits are largely parameter-dependent and subject to frequent revisions. References for the BFRT, BFR, telescope, cavity, solar and red giant limits are [42, 24], 18, 41], [11 13], 39], 37, respectively.

\section{Figure 2.}

Time of solar alignment during one year for fixed horizontal magnet orientations at CERN: (a) for a completely stationary magnet, (b) for $\pm 1.5^{\circ}$ tracking of the solar core in all directions around the otherwise stationary magnet axis orientation, and (c) for $\pm 5^{\circ}$ tracking in all directions around the otherwise stationary magnet axis orientation.

\section{Figure 3.}

Detailed limits on the coupling strength of axions to two photons as a function of the axion rest mass : The top figure corresponds to a $10 \mathrm{~m} \mathrm{LHC}$ test magnet with $\pm 5^{\circ}$ vertical tracking and $\pm 40^{\circ}$ horizontal tracking, the bottom figure is for two such magnets in series with an increased vertical tracking of $\pm 10^{\circ}$. (a) After 1 y with vacuum in the pipe line, (b) an additional $1 \mathrm{y}$ with He gas pressure increased from 0-1 atm in 100 increments, (c) an additional 1 y with 1-10 atm in 365 increments, (d) previous limits from ref. [24], (e) limits recently imposed by members of this collaboration using an underground Ge detector [32], (f) the theoretical red giant limit [37], (g) recent limits from the Tokio axion helioscope [34,38 and (h), the new helioseismological constraints on solar axion emission [39]. The asymptotic behaviour for large axion masses in (d) is a conservative approximation [40]. The "telescope search" limits arise from the absence of an axion-decay quasi-monochromatic photon line from galactic clusters [18,41.

\section{Figure 4.}

Region of the sky (dotted) in galactic coordinates (the galactic center (GC) is at $b=0^{\circ}$, $l=0^{\circ}$, the galactic plane at $b=0^{\circ}$ ), scanned by the suggested axion telescope during 1998 for a) a magnet with $\pm 5^{\circ}$ vertical and $180^{\circ}$ horizontal tracking and, b) the same magnet with a horizontal tracking range limited to NE-SE (enough to track sunset and sunrise during the whole year). It is evident that a horizontal tracking as large as possible is desirable to explore regions of interest such as the GC. For modest vertical tracking, regions of the sky with $|\delta|>90^{\circ}-|\phi|$, where $\delta$ is the equatorial declination and $\phi$ is the magnet's geographical latitude $\left(46.25^{\circ} \mathrm{N}\right.$ for CERN), cannot be explored 
regardless of horizontal tracking ability [25]. The present epoch equatorial coordinates of GC are $\alpha=265.5^{\circ}$ and $\delta=-28.9^{\circ}$, while the galactic north pole is at $192.2^{\circ}$ and $27.4^{\circ}$, respectively. 
[1] S. Weinberg, Phys. Rev. D11 (1975) 3583.

[2] R.D. Peccei, H.R. Quinn, Phys. Rev. D16 (1977) 1791; Phys. Rev. Lett. 38 (1977) 1440.

[3] F. Wilczek, Phys. Rev. Lett. 40 (1978) 279; S. Weinberg, ibid 48 (1978) 223.

[4] J. Ellis, CERN-TH/96-10.

[5] G. G. Raffelt, Proc. XV Moriond Workshop, Dark Matter, Clocks and Tests of Fundamental Laws, Villars-sur-Olon, Switzerland (Jan. 1995); HEP-PH/9502358.

[6] P. Sikivie, Int. J. Mod. Phys. D3 S (1994) 1 and Phys. Rev. Lett. 51 (1983) 1415.

[7] S. L. Cheng, C. Q. Geng, W.-T. Ni, NUCU-HEP-94-20 and hep-ph/9506295.

[8] P.F. Smith and J.D. Lewin, Phys. Rep. 187 (1990) 203.

[9] G.G. Raffelt, "Stars as laboratories for fundamental physics", The University of Chicago Press, Chicago and London (1996), ch. 5 and 14.

[10] P. Sikivie, Phys. Today 49 (1996) 22, HEP-PH/9506229.

[11] K. van Bibber, et al., Int. J. Mod. Phys. D3 S (1994) 33.

[12] C. Hagmann, et al., Phys. Rev. Lett 80 (1998) 2043.

[13] I. Ogawa, S. Matsuki, K. Yamamoto, Phys. Rev. D53 (1996) 1740.

[14] Y. K. Semertzidis, et al., Nucl. Instr. Meth. A356 (1995) 122.

[15] G. Raffelt, A. Weiss, Phys. Rev. D51 (1995) 1495.

[16] S. V. Krasnikov, Phys. Rev. Lett. 76 (1996) 2633.

[17] H.-T. Janka, et al., Phys. Rev. Lett. 76 (1996) 2621.

[18] M. T. Ressell, Phys. Rev. D44 (1991) 3001.

[19] T. W. Kephart and T. J. Weiler, Phys. Rev. D52 (1995) 3226.

[20] K. van Bibber, et al., Phys. Rev. D39 (1989) 2089.

[21] G. Raffelt and L. Stodolsky, Phys. Rev. D37 (1988) 1237.

[22] E. Masso and R. Toldra, Phys. Rev. D52 (1995) 1755, J. A. Grifols, E. Masso, R. Toldra, Phys. Rev. Lett. 77 (1996) 2372, E. Masso, ASTRO-PH/9704056 and J. W. Brockway, E. D. Carlson, G. G. Raffelt, Phys. Lett. B383 (1996) 439.

[23] D. B. Kaplan, Nucl. Phys. B260 (1985) 215. 
[24] D. M. Lazarus, G. C. Smith, R. Cameron, A. C. Melissinos, G. Ruoso, Y. K. Semertzidis, F. A. Nezrick, Phys. Rev. Lett. 69 (1992) 2333.

[25] F. Hoogeveen and R. Stuart, Phys. Lett. B286 (1992) 165.

[26] P. V. Vorobyov, I. K. Kolokolov, ASTRO-PH/9501042.

[27] S. Moriyama, Phys. Rev. Lett. 75 (1995) 3222.

[28] W. Keil, et al., Phys. Rev. D56 (1997) 2419.

[29] J. Morales, E. Garcia, A. Ortiz de Solorzano, A. Morales, R. Nunez-Lagos, J. Puimedon, C. Saenz, J. A. Villar, Nucl. Instr. Meth. A321 (1992) 410.

[30] R. L. Brodzinski, H. S. Miley, J. H. Reeves, F.T. Avignone III, J. Radioanal. Nucl. Chem. 193 (1995) 61; H. S. Miley, R. L. Brodzinski, J. H. Reeves, ibid 160 (1992) 371; R. L. Brodzinski, J. H. Reeves, F. T. Avignone III, H. S. Miley, ibid 124 (1988) 513.

[31] G. Heusser, Annu. Rev. Nucl. Part. Sci. 45 (1995) 543.

[32] F. T. Avignone III, D. Abriola, R. L. Brodzinski, J. I. Collar, R. J. Creswick, D. E. DiGregorio, H. A. Farach, A. O. Gattone, F. Hasenbalg, H. Huck, H. S. Miley, A. Morales, J. Morales, S. Nussinov, A. Ortiz de Solozano, J. H. Reeves, D. Tomasi, I. Urteaga, J. A. Villar and K. Zioutas. Phys. Rev. Lett., in press (1999).

[33] Y. Inoue, S. Moriyama, H. Hara, M. Minowa and F. Shimokoshi, Nucl. Instr. and Meth. A368 (1996) 556.

[34] S. Moriyama, Ph. D. dissertation, University of Tokio, 1997.

[35] M. Bona, et al., "Performance of the first CERN-INFN $10 \mathrm{~m}$ Long Superconducting Dipole Prototype for the LHC", Proc. of the 4th European Particle Accelerator Conference, London (June 1994) 2289.

[36] J. Billan, et al., "Design and Test of the Benches for the Magnetic Measurements of the LHC dipoles", Proc. of the 13th International Conference on Magnet Technology", Victoria, Canada (Sept. 1993).

[37] G.G. Raffelt, Phys. Rev. D33 (1986) 897; G.G. Raffelt and D. Dearborn, Phys. Rev. D36 (1987) 2211.

[38] S. Moriyama, M. Minowa, T. Namba, Y. Inoue, Y. Takasu and A. Yamamoto, Phys. Lett. B434 (1998) 147.

[39] H. Schlattl, A. Weiss and G. Raffelt, HEP-PH/9807476 (submitted to Astrop. Phys.).

[40] Y. K. Semertzidis, private communication.

[41] M. Bershady, et al., Phys. Rev. Lett. 66 (1991) 1398.

[42] R. Cameron et al., Phys. Rev. D47 (1993) 3707; Y.K. Semertzidis et al., Phys. Rev. Lett. 
64 (1990) 2988. 


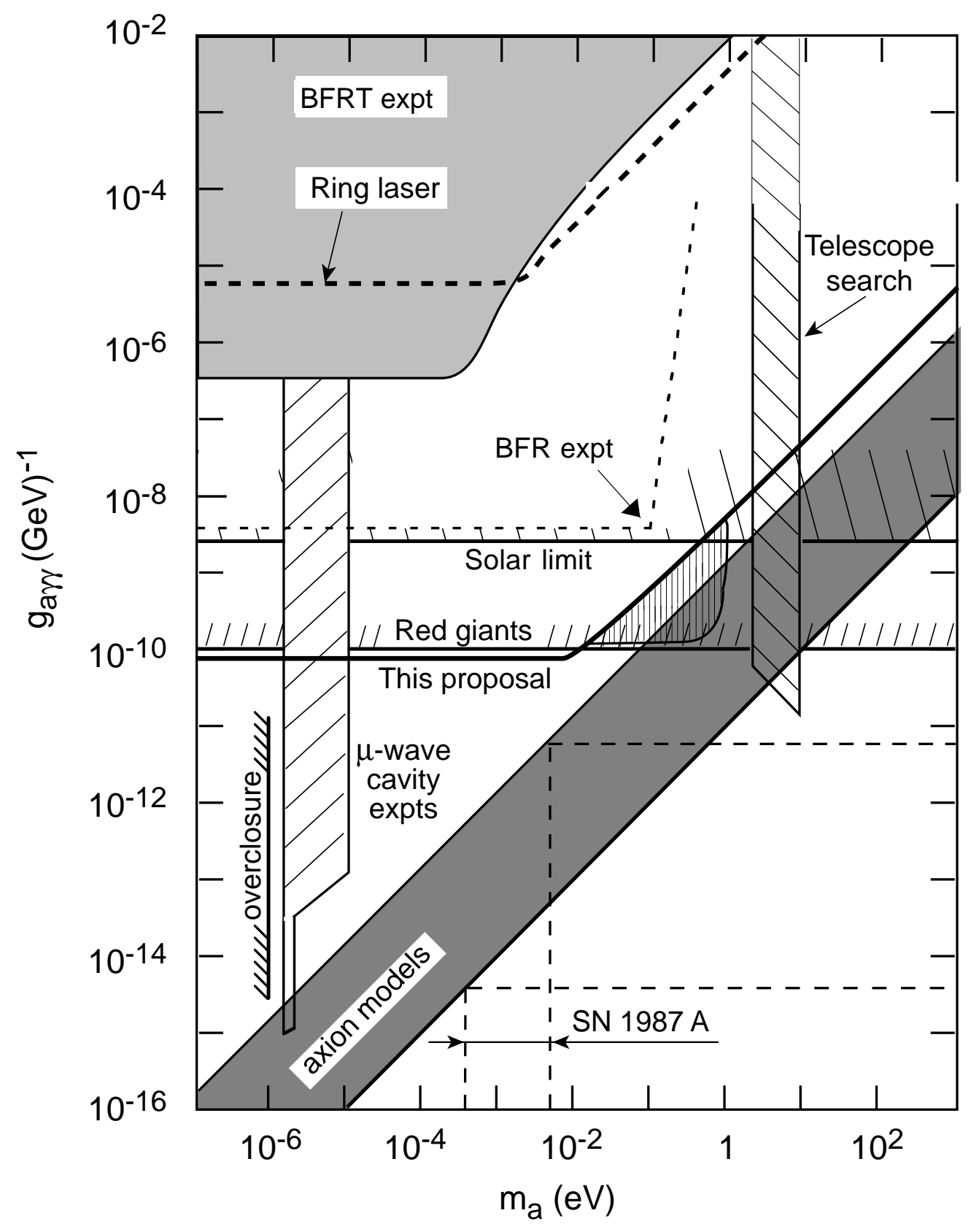

Fig. 1 
a )

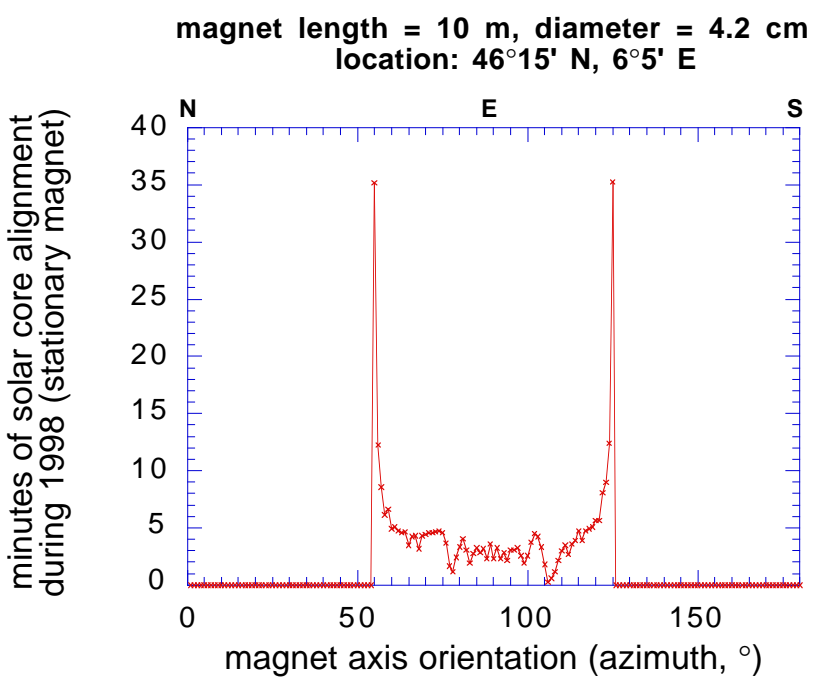

b )

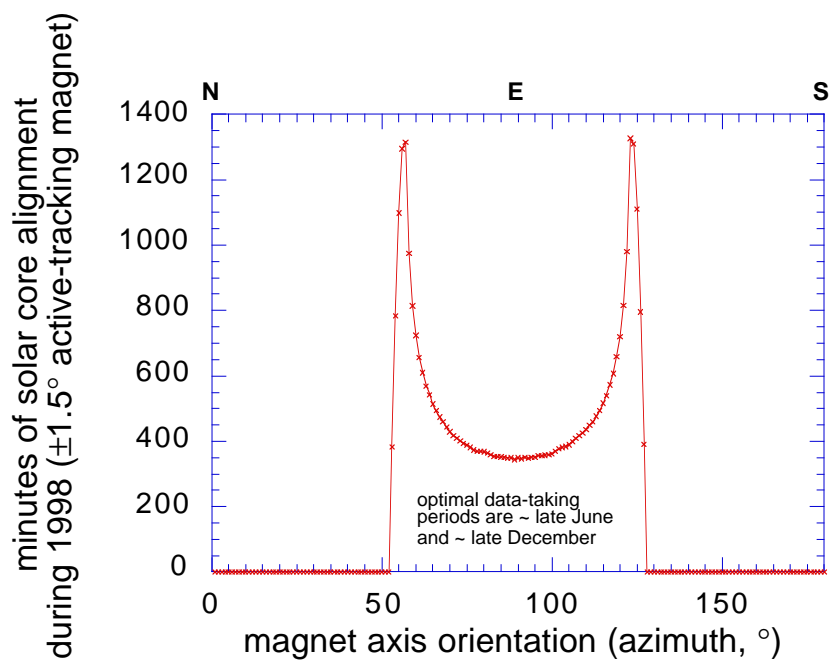

c )

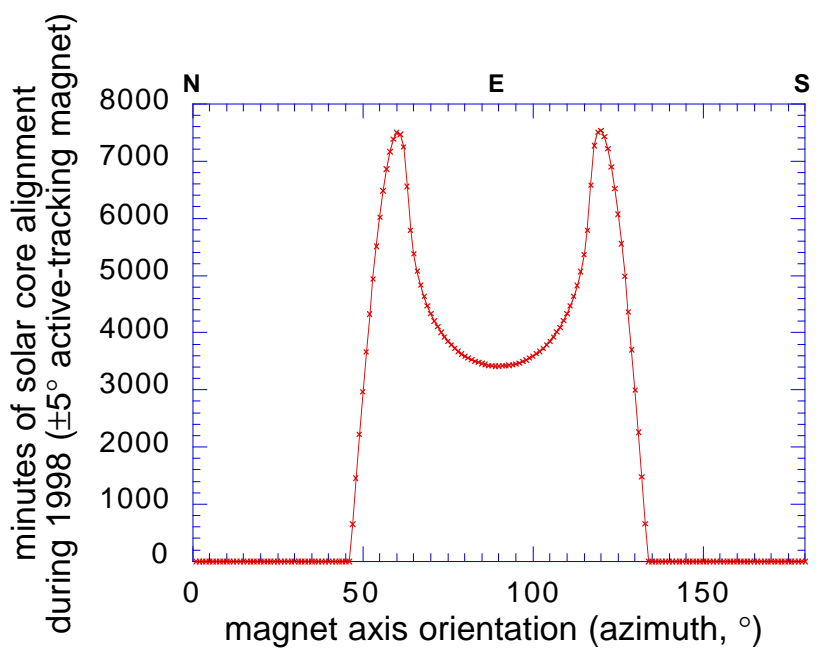

Fig. 2 


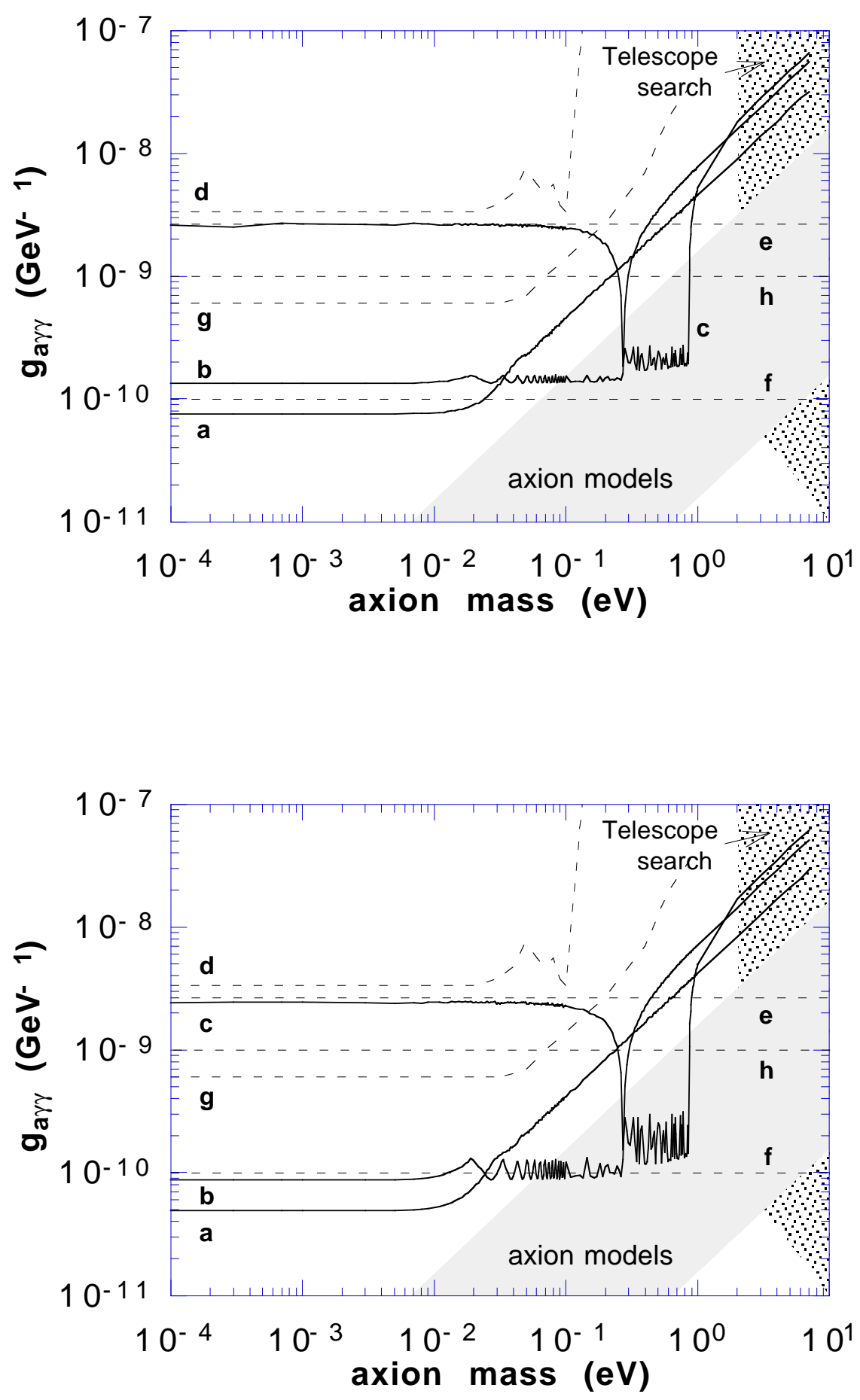

Fig. 3 
a )

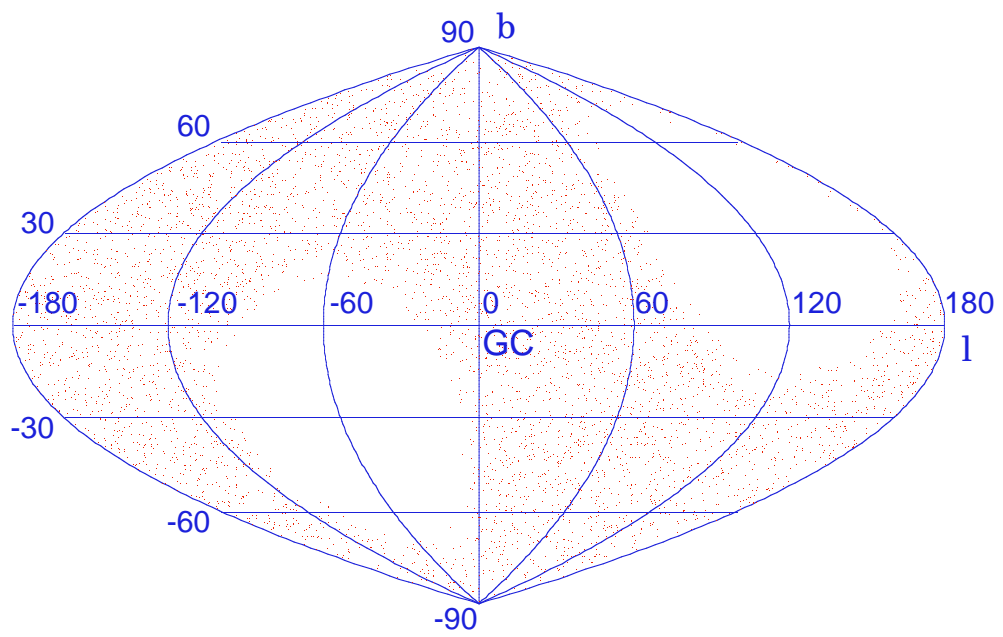

b )

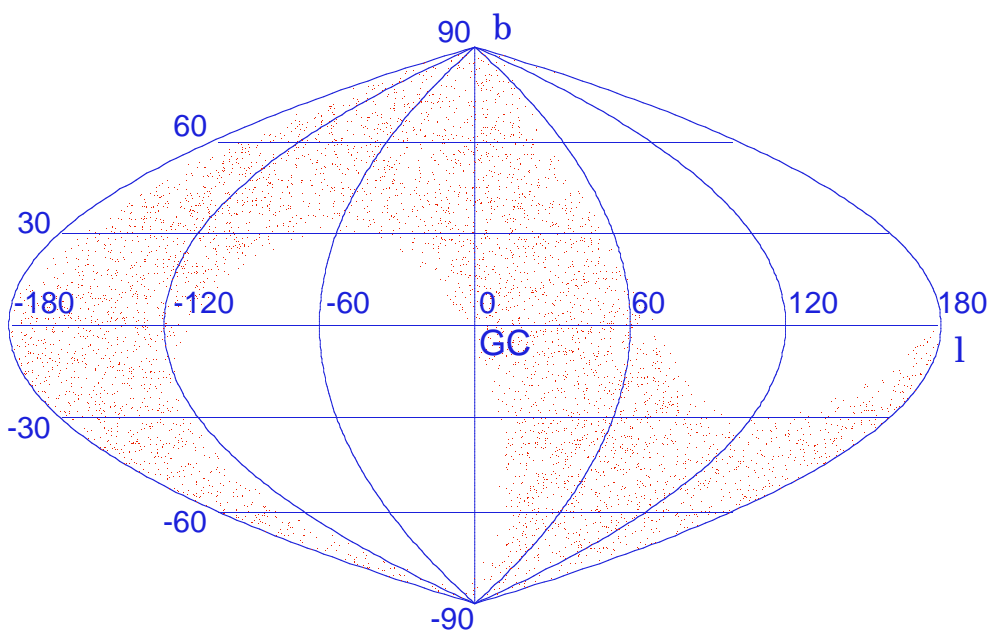

Fig. 4 